\title{
Collective stochastic resonance in shear-induced melting of sliding bilayers
}

\author{
Moumita Das, ${ }^{1, *}$ G. Ananthakrishna, ${ }^{1,2, \dagger}$ and Sriram Ramaswamy ${ }^{1, \dagger}$ \\ ${ }^{1}$ Centre for Condensed Matter Theory, Department of Physics, Indian Institute of Science, Bangalore 560 012, India \\ ${ }^{2}$ Materials Research Centre, Indian Institute of Science, Bangalore 560 012, India
}

\begin{abstract}
The far-from-equilibrium dynamics of two crystalline two-dimensional monolayers driven past each other is studied using Brownian dynamics simulations. While at very high and low driving rates the layers slide past one another retaining their crystalline order, for intermediate range of drives the system alternates irregularly between the crystalline and fluidlike phases. A dynamical phase diagram in the space of interlayer coupling and drive is obtained. A qualitative understanding of this stochastic alternation between the liquidlike and crystalline phases is proposed in terms of a reduced model within which it can be understood as a stochastic resonance for the dynamics of collective order parameter variables. This remarkable example of stochastic resonance in a spatially extended system should be seen in experiments which we propose in the paper.
\end{abstract}

\section{INTRODUCTION AND RESULTS}

\section{A. Background}

The shear flow of a solid is one of the most important and widely studied [1-4] nonequilibrium phenomena in materials science, with relevance to practical problems such as the yielding of materials, solid friction, and even the mechanical properties of the Earth's crust. Such flow takes place when solids are subjected to stresses which range from a few percent of the shear modulus to, in some cases, a value of the order of the shear modulus itself. It is particularly convenient to study such phenomena using very soft solids, where the desired stress to modulus ratio is easily achieved. Indeed, such studies open up new regimes in the physics of driven systems. A variety of such unconventional, ultrasoft solids have been studied, including packings of multilamellar vesicles [5], vortex lattices in type-II superconductors [6], and crystalline arrays, electrostatically or sterically stabilized, of colloidal particles in aqueous suspensions [7]. Experiments on suspensions of interacting colloidal particles under shear are of particular interest to us here, for the rich range of interesting phenomena they reveal, including the shear-induced distortion of the static structure factor in the fluid state, and stick-slip dynamics [8], hysteresis [9], and shear-induced melting $[10,11]$, in the crystalline state. It is likely that the properties of sheared crystals, as observed in macroscopic three-dimensional scattering studies or in timeor frequency-domain mechanical measurements, are the average result of many intermittent, spatially inhomogeneous internal events. Accordingly, this paper focuses on such events, at the level of the relative motion of an adjacent pair of layers, since we believe that knowledge of these events will greatly aid our understanding of the mechanisms underlying phenomena such as shear melting. We emphasize at the outset, to avert any confusion on this score, that the phenomena which our study uncovers, and which we discuss in de-

\footnotetext{
*Email address: moumita@physics.iisc.ernet.in

†Email address: garani@mrc.iisc.ernet.in

"Email address: sriram@physics.iisc.ernet.in
}

tail below, are quite distinct from the well-known stick-slip effect in atomically thin fluid films subjected to shear [12-15].

One popular approach to the study of sheared solids has been to consider an ordered layer (the adsorbate), dragged over a fixed, rigid periodic potential (the substrate), the latter representing an adjacent layer [16-18]. This description is clearly limited in its applicability since it rules out deformation of the substrate, although it is a reasonable starting point for experimental situations in which the overlayer is much softer than the substrate. It is natural, and more general, to ask instead what happens when both adsorbate and substrate are dynamical, and organize themselves into various structures, depending on interaction strengths, temperature and driving force, and it is in this spirit that our model is formulated. The case where both layers are comparably deformable, in particular, is clearly of relevance to sheared crystals. In all cases, each layer confronts a periodic potential produced by the other layer, but both amplitude and phase of this periodic potential are dynamical and change as a result of interactions, noise, and driving force, giving rise to some remarkable collective effects, reported briefly earlier [19] and discussed in detail in this paper.

Although the primary motivation for this paper was the problem of sheared colloidal crystals, there are two other classes of problems to which our study has a natural connection. One is the phenomenon of lane formation in counterdriven interacting particles [20], the other is the equilibrium modulated-liquid to solid transition of interacting particles in an external periodic potential. We will touch upon the relation of these problems to our work later in this paper.

\section{B. Summary of models and results}

We report two detailed studies in this paper: first, a Brownian dynamics simulations of a many-particle model [19], henceforth referred to as the particle model, and second, a reduced model, introduced to get insight into the results of the particle model, consisting of just two degrees of freedom [21], an order parameter amplitude, and a strain field. The particle model consists of two species of particles, 


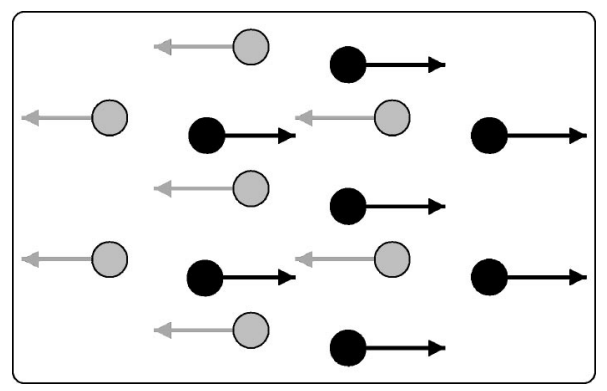

FIG. 1. Schematic diagram of the model

$A$ and $B$, driven by a force $F$, of constant magnitude, in opposite directions, say, along $+x$ and $-x$, respectively (Fig. 1). The $V_{A A}$ and $V_{B B}$ interactions are identical. The $V_{A B}$ interaction has the same form but is smaller by a factor $\epsilon$. This factor, in a phenomenological way, incorporates the physics of the third direction (see below). All pairwise interactions are of screened Coulomb form, with the screening parameter so chosen that, when $F=0$, each species in the absence of the other settles down in a macroscopically ordered triangular lattice configuration. The dynamics of the system is modeled by the overdamped Langevin equation for relatively sheared sets of particles and is monitored for different $F$ and $\epsilon$. With the interlayer coupling strength $\epsilon$ held constant, on increasing the drive, we observe an interesting sequence of nonequilibrium states, namely, a sliding crystalline ordered state (Fig. 2), a sliding melt-freeze state (characterized by alternate states of order and disorder in time), followed again by a sliding ordered state. In the intermediate "melt-freeze" regime, for fixed drive, the residence time of the system in the ordered state decreases and that of the disordered state increases as a function of $\epsilon$ (Figs. 3-5). The allowed nonequilibrium states are best understood in terms of a dynamic phase diagram of these states. We present such a dynamical nonequilibrium phase diagram (Fig. 10) demarcating the three regimes (i) lower smooth sliding, (ii) alternating melt-freeze state, and (iii) upper smooth sliding state. The melt-freeze alternations are most pronounced in a window of driving force $F$ and interlayer coupling $\epsilon$ values. These melt-freeze cycles are strongly reminiscent of the time series of a system undergoing stochastic resonance [22-25] and, to explore this aspect in more detail following Ref. [21],
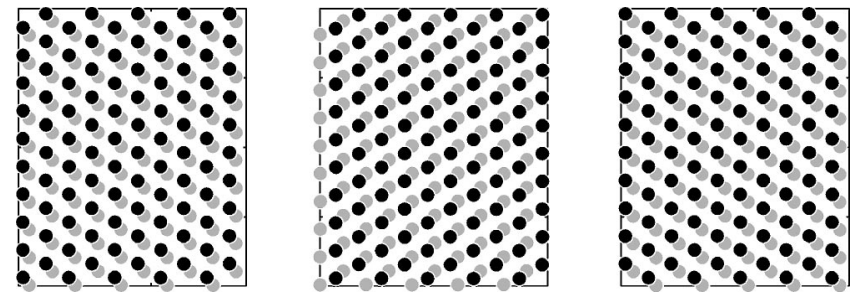

FIG. 2. Simulation images of macroscopically ordered lattices drifting through each other for $\epsilon=0.05$ and $F_{d}^{*}=0.0438$.

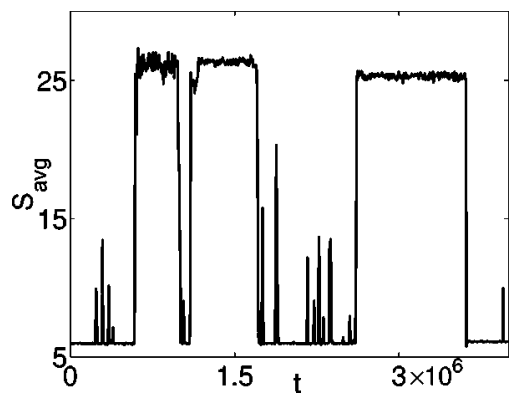

FIG. 3. The structure factor height (averaged over the first ring of maxima) as a function of time in the melt-freeze cycle state for $\epsilon=0.05$ and $F_{d}^{*}=0.8167$.

we introduce the reduced model. Using the reduced model, we study the time evolution of the system using coupled time-dependent Ginzburg-Landau equations for the order parameter and strain fields, as a function of a coupling parameter $\alpha$ entering the model equations and a drive $\Omega$ analogous to $\epsilon$ and $F$, respectively. For a certain range of values of $\alpha$, keeping $\alpha$ fixed, as a function of the drive parameter $\Omega$, we observe three regimes (Figs. 11 and 12) - a crystalline state (nonzero order parameter value), a bistable regime where the system alternates between the crystalline and liquid state (order parameter values being zero), followed again by a crystalline state. Keeping $\Omega$ fixed at an optimum value, we find that the ratio of the average lifetime of the crystalline state to that of the liquid state in the intermediate regime of bistability decreases as $\alpha$ is increased (Figs. 13 and 14). These observations are remarkably similar to the phenomenon observed in the particle model and indeed the phase diagrams of the two models (Figs. 10 and 17) correspond surprisingly well. Further, the reduced model exhibits a maximum in the signal-to-noise ratio at optimum values of the noise intensity (Fig. 16), thereby making the connection to stochastic resonance concrete [22-25].

The paper is organized as follows. The Brownian dynamics simulations of the particle model are described in Sec. II A and the results discussed in detail in Sec. II B. This is followed by physical arguments in support of the behaviors observed. The reduced model [21] is introduced in Sec. III A and its results discussed in Sec. III B. Finally, in Sec. IV we provide a discussion of our results, suggest how our obser-

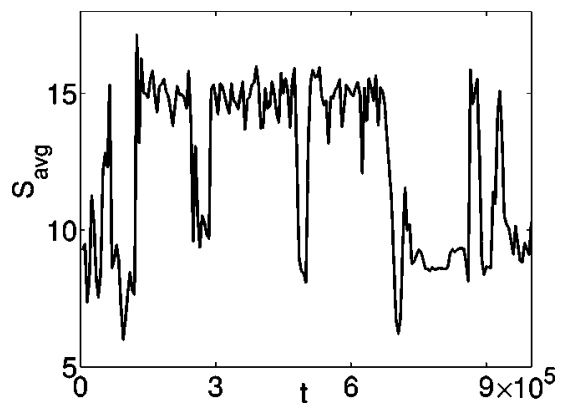

FIG. 4. The structure factor height (averaged over the first ring of maxima) as a function of time in the melt-freeze cycle state for $\epsilon=0.02$ and $F_{d}^{*}=0.8167$. 


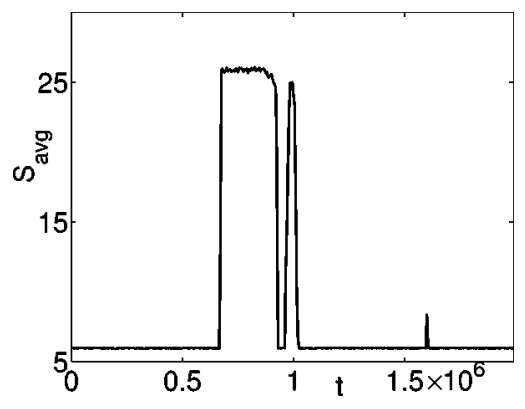

FIG. 5. The structure factor height (averaged over first ring of maxima) as a function of time in the melt-freeze cycle state for $\epsilon$ $=0.06$ and $F_{d}^{*}=1.4350$.

vations may be verified experimentally, and outline directions of future research.

\section{BROWNIAN DYNAMICS SIMULATIONS OF TWO ADJACENT MONOLAYERS}

\section{A. Particle model}

We consider two sets $A$ and $B$ of Brownian particles in two spatial dimensions, driven in the $+x$ and $-x$ directions respectively by a constant driving force with magnitude $F$ as shown in Fig. 1. Pairwise interactions between particles are described by potentials $V_{A A}(r), V_{B B}(r)$, and $V_{A B}(r)$. We choose a rectangular box of dimensions $L=(\sqrt{3} / 2) \times 20 \ell$ and $W=20 \ell$, where $\ell=\left(2 \sqrt{3} n_{0}\right)^{-1 / 2}, n_{0}$ being the mean number density of either species. All quantities we use are in nondimensional form. Lengths are nondimensionalized by $\ell$ and time by $\tau \equiv \ell^{2} / D, D$ being the Brownian diffusivity. Energy is scaled by $k_{B} T$ and force by $k_{B} T / \ell$, where $T$ is the temperature and $k_{B}$ the Boltzmann constant. If the two close packed planes, with species $A$ and $B$ in distinct planes, are slid past each other, it is highly plausible that $V_{A B}$ will be weaker than $V_{A A}$ and $V_{B B}$ (since the effect of the interlayer interaction on the two-dimensional in-plane motion of the particles, which is what $V_{A B}$ encodes, is substantially smaller than that due to the intralayer interaction) and that the strength of $V_{A B}$ relative to $V_{A A}$ and $V_{B B}$ can be varied by increasing or decreasing the normal confining pressure. We therefore choose for this work dimensionless pair potentials of the screened Coulomb form,

$$
V_{A A}(r)=V_{B B}(r)=\epsilon^{-1} V_{A B}(r)=\left(U_{0} / r\right) \exp (-\kappa r),
$$

where $U_{0}\left(=1.75 \times 10^{4}\right)$ and the screening parameter $(\kappa \ell$ $=0.5$ ) are so chosen that each species in the absence of the other and any external driving force settles down in a triangular lattice configuration (Fig. 1). The particle positions then evolve according to the overdamped Langevin equations,

$$
\mathbf{R}_{\mu}^{i}(t+\delta t)=\mathbf{R}_{\mu}^{i}(t)+\delta t\left[\mathbf{F}_{\mu}{ }^{i}+\mathbf{f}_{\mu}^{i}\left(\mathbf{R}_{\mu}(t)\right)+\mathbf{h}_{\mu}^{i}(t)\right],
$$

where $R_{\mu}^{i}=\left(x_{i \mu}, y_{i \mu}\right)$ is the coordinate of the $i$ th particle of the species $\mu(=A$ or $B)$, and $\pm F \hat{x}$ ( + for $A,-$ for $B$ ) is the external driving force on the $i$ th particle of type $\mu$.

$$
\mathbf{f}_{\mu}^{i}\left(\mathbf{R}_{\mu}^{i}\right)=-\sum_{j \neq i} \nabla V_{\mu^{i} \nu^{j}}\left(\mathbf{R}^{i}-\mathbf{R}^{j}\right)
$$

are the interparticle forces and $h_{\mu}^{(i)}$ are Gaussian white noise sources with zero mean obeying the fluctuation dissipation relation which in nondimensional form reads

$$
\left\langle\mathbf{h}_{\mu}^{i}(0) \mathbf{h}_{\nu}^{j}(t)\right\rangle=2 \mathbf{I} \delta_{\mu \nu} \delta^{i j} \delta(t)
$$

where $\mathbf{I}$ is the unit tensor. It is trivial to generalize to the case where species $A$ and $B$ differ but we have chosen them to be same in this model. We study the time evolution of this system as a function of the drive keeping $\epsilon$ constant for several values of $\epsilon$. The dimensionless time step used in our simulations is $\delta t=6.4 \times 10^{-6}$.

\section{B. Simulation results}

The results reported in our study are generally for $10^{6}-10^{7}$ time steps after the initial transients $\sim 10^{4}$ steps are discarded. Over this time, the $A$ and $B$ lattices sweep through each other a few to several hundred times depending upon the magnitude of the drive. In order to drift under the action of the driving force $F$, the particles have to overcome a barrier of the order of $V_{A B}(\ell)$ arising from interaction with the nearest neighbors of the opposite species. Thus, although $F$ is itself dimensionless, it is appropriate to state the results in terms of the physically relevant dimensionless combination $F_{d} \equiv F \ell / V_{A B}(\ell)$. However, for the phase diagram in the $\epsilon-F$ variables, we have used the dimensionless combination $F_{d}^{*}$ $\equiv F \ell \epsilon / V_{A B}(\ell)$, as $V_{A B}$ already incorporates a factor of $\epsilon$ in its definition. The structure and dynamics of the system has been monitored through snapshots of configurations, drift velocities $v_{d}$, particle-averaged local velocity variances $\left\langle(\delta v)^{2}\right\rangle$, pair correlation functions $g_{\mu \nu}(\mathbf{r})$ as functions of separation $\mathbf{r}$, and time-dependent (but equal time) structure factors $S_{\mu \nu}(\mathbf{k}, t)$ as functions of wave vector $\mathbf{k}$ ( $\mu$ and $\nu$ range over $A, B)$. Each point in the $g_{\mu \nu}(\mathbf{r})(\mathbf{r}=x, y)$ is an average over 100 data points, recorded at times separated by $50 \delta t$. In the absence of the driving force (i.e., at $F_{d}=0$ ), the system is an imperfectly ordered crystal. The application of a small nonzero $F_{d}$, well below the apparent threshold for perceptible macroscopic relative motion of the two lattices (of $A$ and $B$ particles, respectively), facilitates particles that are initially in unfavorable positions to reorganize and move to favorable locations leading to a small movement in these regions. After these transient motions the system settles down into a macroscopically ordered structure with both components showing perfect long-range crystalline order sustained over distances of the order of the system size. There is no further relative drift of $A$ and $B$ except perhaps a tiny activated creep which we cannot resolve. Thereafter, keeping interaction strengths and temperature fixed, the driving force $F$ displays three threshold values $F_{i}, i=1,2,3$ corresponding to the lower bounds of three states-a lower sliding crystalline state, a melt-freeze state, and an upper sliding crystalline state. The characteristic features of each of these three states are mentioned below. 


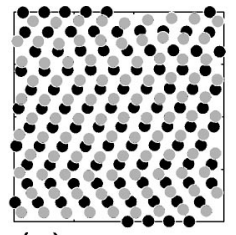

(a)

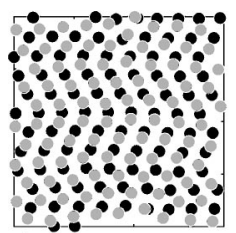

(c)

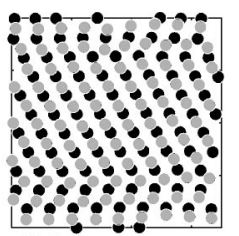

(b)

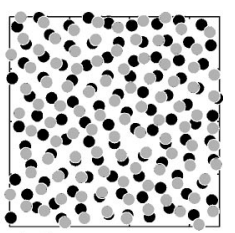

(d)
FIG. 6. Particle configuration snapshots at the onset of disorder in the melting-freezing regime for $\epsilon=0.05$ and $F_{d}^{*}=0.8167$.

When $F_{d}$ crosses the first threshold $F_{1}$, the $A$ and $B$ components acquire a measurable, macroscopic relative drift velocity $v_{d}$. The drift velocity shows a smooth change at this threshold value with the velocity fluctuations [26] showing a pronounced enhancement [19] characteristic of depinning. (See Figs. 3 and 4 of Ref. [19].) This is likely to be a strong crossover rather than a true transition. Each particle faces a finite barrier to motion, so that at any nonzero temperature, particles can cross the barrier individually in an incoherent manner for arbitrarily small $F_{1}$. The barrier for creep velocity should thus be finite even in the limit of infinite system size. In the region $F_{1}<F<F_{2}$, both $A$ and $B$ components are well-ordered, drifting crystals (Fig. 2). The two components slide smoothly past each other in lanes of width equal to the interparticle distance, with negligible distortion or disorder. We comment later on the connection to the lane formation work of Ref. [20].

In a window of driving forces $F_{2}<F<F_{3}$, we observe intriguing stochastic alternations of the system between an

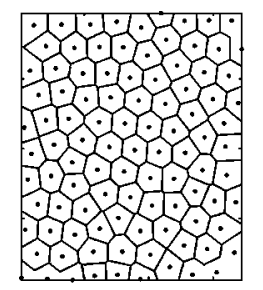

At $5.8 \times 10^{5}$ timesteps

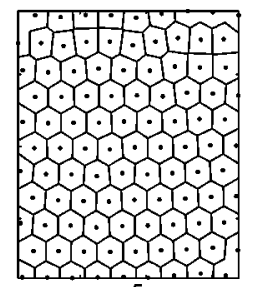

At $9.8 \times 10^{5}$ timesteps

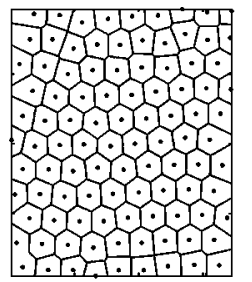

At $5.85 \times 10^{5}$ timesteps

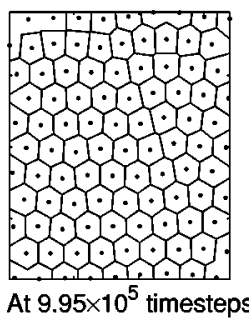

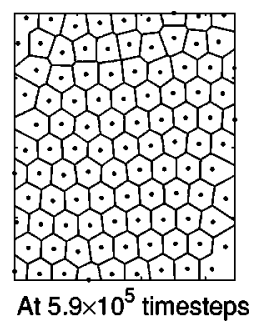

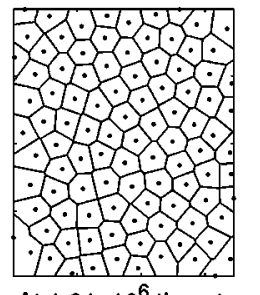

FIG. 7. Voronoi construction from particle configurations showing the onset of order and of disorder for species $A$ at $\epsilon=0.05$ and $F_{d}^{*}=0.8167$.
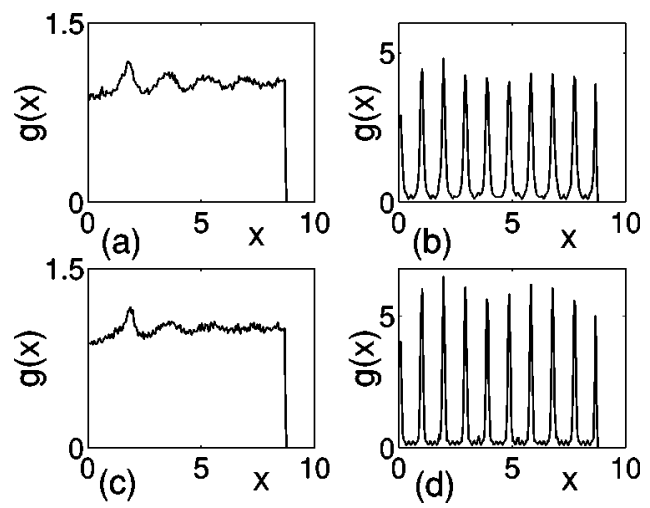

FIG. 8. The distribution function along $x$ direction for species $A$ for $\epsilon=0.05$ and $F_{d}^{*}=0.8167$.

ordered and a disordered state which are the melt-freeze cycles (Figs. 4, 3, and 5). We have used snapshots of configurations shown in Figs. 6 and 7, the pair correlation function $g_{\mu \nu}(\mathbf{r}), \mathbf{r}=x, y$ (Figs. 8 and 9), and the equal-time but time-dependent structure factor $S(\mathbf{k}, t)$ to characterize these dynamical states of order and disorder. As the first two methods are representative of the instantaneous state of the system, for monitoring these cycles continuously we use the peak height of the (short-time averaged) static structure factor $S(\mathbf{k}, t)$. Indeed, the essential features of these cycles, namely the persistence duration, fluctuations in the extent of order, etc, are best captured through the time dependence of $S(\mathbf{k}, t)$ as we shall see. A typical such plot is shown in Fig. 3 for an optimum value of $\epsilon=0.05$. It is clear that $S(\mathbf{k}, t)$ alternates between long stretches of crystal-like [corresponding to large values of $S(\mathbf{k}, t)$ ] and comparably long stretches of liquidlike values [small values of $S(\mathbf{k}, t)$ ] as the simulation progresses. This is strikingly different from stick-slip alternations, in which the melted (slip) state is considerably shorter than the crystalline (stick) state $[12,13]$. We discuss this comparison later in Sec. IV. For smaller values of $\epsilon$, even as the persistence of the crystalline state is enhanced, the extent of ordering itself is less pronounced as indicated by the lower values of $S$ (compared to that for the crystalline phase corresponding to $\epsilon=0.05)$. Concomitantly, the liquidlike state also displays significant short-range order. These
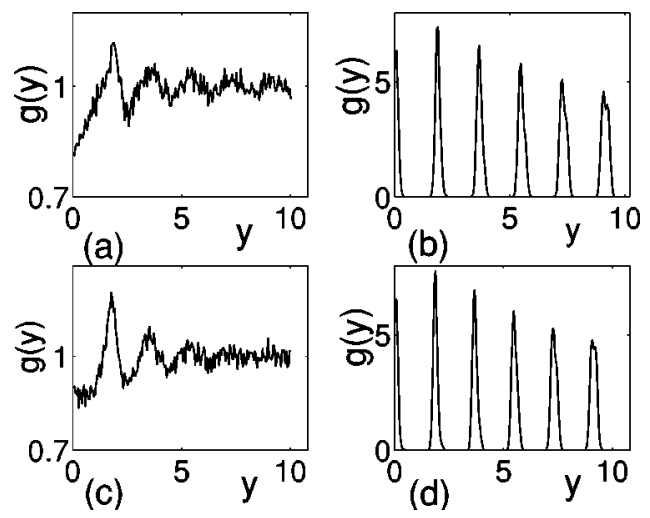

FIG. 9. The distribution function along $y$ direction for species $A$ for $\epsilon=0.05$ and $F_{d}^{*}=0.8167$. 
features are clear from Fig. 4 where $S(\mathbf{k}, t)$ is shown for $\epsilon$ $=0.02$. For higher values of $\epsilon$, the liquidlike state is more favored (as can be seen from the short stretches of crystalline order) for $\epsilon=0.06$ shown in Fig. 5 .

As is clear from Figs. 3-5, the time scales over which the crystalline order or liquidlike disorder sets in is considerably shorter than the persistence time of each of these phases. The process of ordering and disordering is better monitored through a sequence of snapshots of configurations. A typical set of snapshots is shown in Fig. 6. The corresponding Voronoi construction for $\epsilon=0.05$ is shown in Fig. 7. (See also Fig. 5 in Ref. [19].) The extent of order in the $x$ and $y$ directions is studied using the correlation function. The nature of $g(x)$ and $g(y)$ is shown in Figs. 8 and 9 at four different times. Note that the extent of order in the liquidlike state in the direction of the drive $x$ is significantly less than that in the $y$ direction. Further, the primary ordering wave vectors are along $\hat{x}$ and $60^{\circ}$ to $\hat{x}$. Thus nearest neighbor distance in the $y$ direction is $\sqrt{3}$ times larger. A partial understanding of why order starts to set in again after melting has occurred is to note that relative motion disrupts primarily those structures with ordering wave vector along the drift direction $\hat{x}$, leaving some residual order along $\hat{y}$ as seen in Figs. 8 and 9 . So each species still provides a weak periodic potential along $y$ for the other species. This can induce order along $x$ as well resulting in a two-dimensional (2D) ordered state by a mechanism similar to the "laser-induced freezing" of a 2D suspension of strongly interacting colloidal particles subject to a $1 \mathrm{D}$ periodic modulation [27-30]. This state persists for a long time, before disorder once again sets in. And it is in this driving force regime that one sees the meltingfreezing cycles. Finally, we find that the two species do not necessarily order or disorder simultaneously. Though we found no clear trend in this regard, mostly, one species begins to order while the other is disordered. Also, for both species, the "Bragg peak heights" rise fast and decline slowly, i.e., ordering takes place much faster than the progression of disorder.

The structure factor can be used to obtain the dynamical phase diagram in the $F_{d}^{*}-\epsilon$ plane. This is shown in Fig. 10. The points shown here actually represent the value of $\left(F_{d}^{*}, \epsilon\right)$ for which the crystalline phase is detected just before entering the bistable melt-freeze region (region II). The regions I and III refer, respectively, to the lower and upper sliding crystalline states. We emphasize here that the transition to the melt-freeze regime occurs over a finite range of values of the parameters and it is not possible to pin down with precision the point at which the melt-freeze alternations begin. The range of values of the force, $F_{3}-F_{2}$, over which we observe that the melt-freeze cycles increase with $\epsilon$ and hence with $V_{A B}$ as well (Fig. 10). This agrees well with our observation that the average potential barrier that a particle has to negotiate during its motion in the steady sliding state increases with $\epsilon$. Further, for large values of $\epsilon(\epsilon \geqslant 0.05)$, the alternations persist over a very large window of driving forces. For such $\epsilon$ values, we have not been able to detect the upper threshold $F_{3}$ corresponding to the reentrant crystalline state.

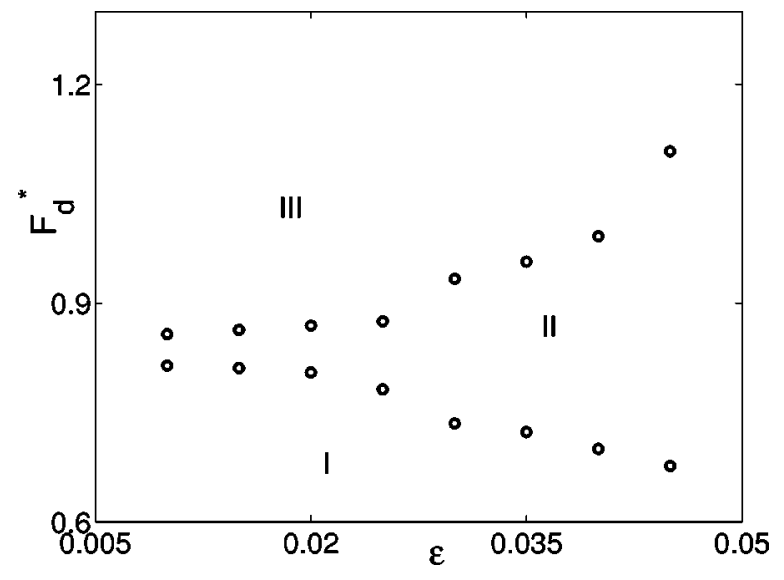

FIG. 10. The phase diagram of the system (100 particles of each species) in the $\epsilon-F_{d}^{*}$ plane, where $F_{d}^{*}=F \ell \epsilon / V_{A B}$. The system undergoes the stochastic melt-freeze alternations in the region II, and is a macroscopically ordered crystal in the upper and lower regions I and III, respectively.

We shall now discuss the melt-freeze cycles in more detail and explain the observed features. There is a curious metastability associated with the cycles: for the parameters mentioned above, a disordered configuration fails to nucleate even over our longest simulations if the initial state is chosen to be a perfectly ordered lattice. Thus, both the melt-freeze cycles and ordered sliding states display local dynamical stability. But if we disturb this initial perfectly ordered lattice by moving a single particle by, say, one lattice spacing, the melt-freeze cycles resume. Also note that the orientation of the triangular lattice in the ordered state of the cycles [Fig. $6(\mathrm{a})]$ is changed by $30^{\circ}$ with respect to the one in the steady sliding state (Fig. 2). This exchange of stabilities between the fcc-like (Fig. 2) and "layer" [Fig. 6(a)] structures is known from experiments [31] and simulations [32]. At low relative velocity, particles in each layer have ample time to get out of the way of those in the other layer while retaining on average the fcc-like structure. As the speed is increased, the structures do not have sufficient time to relax and overlap is reduced by going to the layer structure of Fig. 6(a). However, we observe that even with such a shift in orientation, in the ordered part of the cycle, the smooth relative motion of the $A$ and $B$ lattices is disturbed now and then by kinks; a row moving out of step with adjacent rows, as marked in Fig. 6. This leads to the formation of kinklike undulations transverse to the mean drift like a wave. At some point in time as these undulations build up sufficiently, the system enters a disordered state. This state persists for a long time, before order sets in once again. Recall that at small $\epsilon$, in the ordered part of the cycle, the magnitude of the structure factor is small and correspondingly we find an enhanced level of fluctuations (Fig. 4) compared to that at large $\epsilon$ (Fig. 3). This is because the ordered states at small $\epsilon$ can support a larger number of defects without making a transition to the disordered state, whereas as $\epsilon$ is increased such states cannot be sustained for long time. In fact, with increasing $\epsilon$, the probability of the system being in the ordered state crucially depends on the defect density, i.e., the ordered state is long lived only when the number of defects is small. This can be 
understood by considering the potential landscape seen by each species. In the ordered state, particles of each species are nested in the threefold minima formed by its nearest neighbors of the other species. For small $\epsilon$, both in the lower and upper part of the sliding states, the potential depth is shallow and creation of defects in shallow potentials does not cost much energy. For the same reason, the number of such defects that the state can sustain can also be large, which in turn implies that the extent of order in the crystalline part of the cycles is not significant. A shallow potential also allows for the ease of annealing of the defects as this can be accomplished by removing one particle from a seven-fold coordinated or adding one particle to a five-fold coordinated site (Fig. 7). Thus, low $\epsilon$ situation allows for the ease of creation and annealing out of these defects as time progresses. This dynamical balance between the creations and annihilation of the defects can be sustained for long stretches of time but with smaller extent of order with concomitantly large fluctuations. This is precisely what is seen in Fig. 4. (Note that this picture is also consistent with the observed feature that the liquidlike state at low $\epsilon$ has a fairly high level of order compared to that at high $\epsilon$.) In contrast, with increase in $\epsilon$, the well depth increases significantly which implies that the crystalline order would be high as can be seen in Fig. 3. Moreover, the formation of defects is less favored, but, once formed, it cannot be easily annealed. Further, each defect gives rise to large local restoring forces and the crystalline order will be terminated even when their number is small.

For $F>F_{3}$, both $A$ and $B$ components are once again well-ordered, drifting crystals. In fact, the reappearance of a smooth sliding state is akin to the reentrant ordered state seen in Refs. [21,33].

In fact, the sliding crystalline states that we observe at low and high drives can be visualized as ordered states with lanes [20] of single-particle width. Reference [20] studies a model very similar to ours, with $\epsilon=1$. In that case, the equilibrium state is a crystal, randomly occupied by each species, and the driven state shows the interesting phenomenon of lane formation. Because $\epsilon$ is large, the system tries to minimize the extent of $A B$ interface, hence one gets a few broad lanes with many columns of particles of the same species. Since $\epsilon$ is very small for us, we get many lanes of unit width.

The results stated above are for 100 particles of each species. We have carried out a systematic study of this phenomena for 144, 169, and 256 particles of each type for $\epsilon$ $=0.02$. We find the same qualitative behavior as that for 100 particles including the range of $F_{d}$ values for the three phases. However, for smaller systems (with 64 particles of each species), we have not observed any significant decay in order for $\epsilon<0.1$ (possibly due to the fact that the correlation length is of the order of half the system size in this case).

We now discuss some natural timescales which will be useful in our final explanation of this phenomenon. When the two arrays of particles are driven through each other, there is a competition between two time scales- $\tau_{1}$, the time to traverse one lattice spacing and $\tau_{2}$, the time scale of relaxation of a particle in the local potential well provided by its neighbors. For $\tau_{1} \gg \tau_{2}$, each species has ample time to relax to local equilibrium, while for $\tau_{1} \ll \tau_{2}$, each species averages out the undulating landscape. For both $\tau_{1} / \tau_{2} \gg 1$ and $\ll 1$, we expect and find smooth, orderly sliding. We therefore expect maximal effects of interspecies interaction where $\tau_{1}$ $\approx \tau_{2}$ (for example, for $\epsilon=0.05, \quad \tau_{1} \simeq 0.00022$ and $\tau_{2}$ $\simeq 0.00019$ in the melt-freeze regime [19]), which is what we find. This suggests that a detailed explanation lies in mechanisms involving competing time scales to which we now turn.

The stochastic alternations of the system between the crystalline and liquidlike states is strongly reminiscent of the phenomenon of stochastic resonance (SR) [22-25]. To see the similarities and the differences, consider a prototypical example of stochastic resonance of a Brownian particle in a bistable potential subjected to a weak periodic forcing term. When half the periodicity of the driving force is comparable to the mean first passage time associated with the barrier crossing, the state of the system switches between the two minima of the potential in a surprisingly regular way. Indeed, the time series of the position of the Brownian particle entrained in the two minima is very similar to $S(\mathbf{k}, t)$ of the particle model. What this potential (or an "effective free energy" in this case) is and what modulations are, is not clear in the present model and needs further investigation. One can anticipate that the role of the bistable potential in SR is played by the effective free energy as the system is a manyparticle system switching between the crystalline and liquidlike states. However, identifying a periodic forcing in the present context is more difficult. Thus, it would be useful to construct a reduced model which displays the dominant features of the particle model. One important characteristic feature of SR is that the signal-to-noise ratio exhibits a maximum at an optimum value of the noise intensity. This aspect cannot be easily checked in the particle model as it involves generating very long time series (which would involve prohibitively large scale computing). However, we recall that altering $\epsilon$ in the particle model has an effect that controls the ratio of the residence times of the system in the ordered and disordered states. This is similar to altering the height of the bistable potential which in turn controls the residence time in the example considered. This identification further supports our view that the melt-freeze cycles are, in fact, stochastic resonance. We shall make this more concrete by introducing a reduced model which captures most features of the particle model.

\section{THE REDUCED MODEL}

The effects of external nonequilibrium driving conditions in an underlying first-order phase transition have often been studied successfully by modifying, say, the time-dependent Ginzburg-Landau (TDGL) equation for the dynamics of the order parameter [34]. The results of the preceding section were obtained from direct simulation of particle motion. In this section, we propose an understanding of these results through dynamical equations for the appropriate order parameter fields evolving under the combined effect of shear and a coarse-grained free energy. The nature of the free energy functional is usually determined based on the knowledge of the allowed states of order/disorder and a few 
general symmetry considerations. Here, we follow the model proposed earlier [21] for studying sheared colloidal crystals $[33,35]$. Recall that in our simulations, at low drives, we find a smooth sliding crystal wherein the $A$ lattice slides past that of $B$ in a coherent fashion, and at intermediate drive values, we observe the melt-freeze cycles. To mimic this, we choose an order parameter denoted by $\rho$ (the Bragg peak intensity), which takes on a finite value corresponding to the crystalline order and zero value corresponding to the liquidlike order. A simple form of the free energy which ensures the crystalline $(\rho \neq 0)$ and melt phases $(\rho=0)$ is the Landau polynomial for a first-order transition,

$$
V(\rho)=\frac{a_{1} \rho^{2}}{2}-\frac{b_{1} \rho^{3}}{3}+\frac{c_{1} \rho^{4}}{4} .
$$

The distortions produced due to the drive in the particle model can be represented by another variable representing the strain (in our model, the relative phase of the density wave in the two sliding layers) denoted by $\theta$. In the crystalline state, as distortions are small and homogeneous at low drives, we take $\theta$ to be zero for this state. As homogeneous distortions would mean that the successive minima of the crystal are equivalent, we consider $\theta$ to be a periodic variable with $\theta=0$ to be equivalent to $\theta=1$. Further recall that our simulations show that at intermediate drives, deformation becomes inhomogeneous, forcing the crystalline state to melt (although in a dynamical way). Again, a simple form of the free energy in $\theta$ should incorporate elasticity at small $\theta$ and yielding at large $\theta$, ie., at small strains, $V(\theta)$ is assumed to be quadratic in $\theta$ and a softening term at larger strains (the cubic term). The coefficient of $V(\theta)$ must vanish with $\rho$, say as $\rho^{2}$, as the free energy cost of deformations must reduce to zero when the system is in the liquid state (i.e., for $\rho=0$ ). The simplest general form of the free energy $F(\rho, \theta)$ of a distorted solid respecting the above conditions [21] is of the following form:

$$
F(\rho, \theta)=V(\rho)+\frac{1}{2} \alpha \rho^{2} V(\theta),
$$

where $V(\theta)$ has a similar form as $V(\rho)$,

$$
V(\theta)=\frac{a_{2} \theta^{2}}{2}-\frac{b_{2} \theta^{3}}{3}+\frac{c_{2} \theta^{4}}{4} .
$$

The Langevin-TDGL equations for $\rho$ and $\theta$ that describe the dynamics of this system are

$$
\begin{gathered}
\dot{\rho}=-\frac{1}{\Gamma_{\rho}} \frac{\partial F(\rho, \theta)}{\partial \rho}+\eta_{\rho}, \\
\dot{\theta}=-\frac{1}{\Gamma_{\theta}} \frac{\partial F(\rho, \theta)}{\partial \theta}+\Omega+\eta_{\theta} .
\end{gathered}
$$

The idea is that in the absence of any restoring forces for $\theta$, $\dot{\theta}$ would be equal to $\Omega$. In general, then, $\Omega$ represents the effects of relative sliding of the layers, at a rate determined by competition between $\Omega$ and $\partial F / \partial \theta$. If $\Omega$ is too small, $\theta$ will get stuck at a finite value in the absence of noise. $\Gamma_{q}^{-1}$, $\mathrm{s}$ $(q=\rho, \theta)$ are the kinetic coefficients, $\eta_{q}$ 's represent Gaussian $\delta$-correlated noise components whose variances are related to $\Gamma_{q}$ and temperature through the fluctuationdissipation relation. We ignore possible additional nonequilibrium noise sources.

The equations for our system are those for an overdamped particle in a nonsymmetric double-well potential $F(\rho, \theta)$, driven along the angular coordinate [36]. For zero strain $(\theta$ $=0)$, the system relaxes in either of the two minima corresponding to the liquid minimum,

$$
\rho_{l}=0, \quad \theta=0,
$$

or the crystalline minimum

$$
\rho_{c}=\frac{b_{1}}{2 c_{1}}\left[1+\sqrt{1-\frac{4 a_{1} c_{1}}{b_{1}^{2}}}\right], \quad \theta=0,
$$

depending on which is the locally stable state.

We choose the parameter values $\left(a_{1}, b_{1}\right.$, and $\left.c_{1}\right)$ such that the crystalline minimum $\rho_{c}$ is the more favorable state at zero drive and the potential barrier between the two minima, $V\left(\rho_{l}\right)-V\left(\rho_{c}\right)$, is appropriate. In the presence of noise (whose strength can be appropriately chosen) the system equilibrates with the respective populations determined by noise strength and the relative well depths. If $\theta$ and $\rho$ are macroscopic (infinite system-size) averages, there should be no noise in the equations. In practice, presumably, shear melting and the cycles take place over a finite correlated domain (whose size we do not know). We are therefore justified in using noisy evolution equations.

When the drive $\Omega$ is switched on, this scenario is altered and the populations of each of these wells now evolve in time depending upon the competing time scales of relaxation and applied shear rate. In this case, it is better to consider the fixed points of the noise-free case of Eqs. (8) and (9). The two attractive fixed points to which the system relaxes can now be identified with crystalline and liquidlike order. The repulsive fixed point determines a saddle type of maximum. These will depend on the shear rate $\Omega$. The barrier height between the stable fixed points and unstable fixed point determine the barriers that the system has to surmount. These now depend on $\Omega$. We find that the value of the free energy at the liquidlike minima and the saddle do not change significantly, only the crystalline (distorted) minimum changes as a function of $\Omega$ given by

$$
\rho_{c}=\frac{b_{1}}{2 c_{1}}\left[1+\sqrt{1-\frac{4\left[a_{1}+\alpha V\left(\theta_{\text {min }}\right)\right] c_{1}}{b_{1}^{2}}}\right] .
$$

Note that Eq. (9) determines a critical value of $\Omega=\Omega_{c}$ $=\left.0.5 \alpha \rho^{2}(\partial V / \partial \theta)\right|_{\max }$. For values of $\Omega<\Omega_{c}$, the barrier is high. In such a situation, in the presence of noise, the transitions would be rare. But, on increasing $\Omega$ beyond the critical value the "free energy" of crystalline state becomes comparable with that of the liquid minimum. Under these 


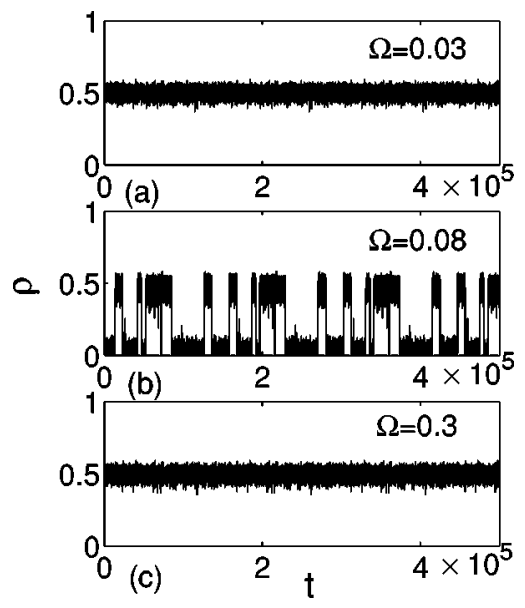

FIG. 11. The time series of the order parameter in the low (top panel), high (bottom panel), and intermediate (middle panel) driving force regimes. We have chosen a noise strength $D=7 \times 10^{-4}$ and coupling parameter $\alpha=0.17$.

conditions, noise assisted transitions to the liquid state occur. More importantly, in this regime, as $\theta$ is itself changing as a function of time, the minima in $(\rho, \theta)$ are slowly modulated under action of the drive $\Omega$ and the relative stability changes as a function of time. When the time scale imposed by $\Omega$ is small enough to allow the system to make interwell transitions and when the time scale of the induced periodicity is approximately equal to the Kramers escape time $[37,38]$ under the influence of noise, one expects transitions between the crystalline and liquidlike states in a range of values of $\Omega$ beyond $\Omega_{c}$. As a result, the system can undergo stochastic transitions between the two metastable states which in turn can lead to comparable lifetimes.

As this is a driven system, a reasonable criterion for studying the occupancy of the system is to calculate the marginal probability distribution function $P(\rho)=\int P(\rho, \theta) d \theta$ (i.e., the probability of the order parameter having the value $\rho$, independent of the value of the strain field $\theta$ ). In the following section we show that the external drive causes the system to sample both minima or stay mainly in one of the minima depending on the value of the drive $\Omega$, noise strength, and coupling constant $\alpha$ starting from an initial crystalline state.

\section{Results of the reduced model}

We study the time series and the probability distribution of the system by discretizing the Langevin equations in time. The integration scheme used is the fourth-order Runge-Kutta with a fixed time step of 0.001. After discarding transients $\left(\sim 10^{5}\right.$ time steps) the time evolution of the system for the next $8 \times 10^{9}$ time steps is monitored. In Figs. 11 and 13 we have shown a time series stretch from $6 \times 10^{9}$ to $6.5 \times 10^{9}$ time steps. Noise corresponding to $\rho$ and $\theta$ are drawn from Gaussian white noise distributions with zero mean and unit variance. For studying the time evolution of the system, we have chosen a noise strength $D=7 \times 10^{-4}$ for both $\eta_{\rho}$ and $\eta_{\theta}$. (We shall study the influence of noise on the signal-tonoise ratio later.) For the numerical work reported here, the

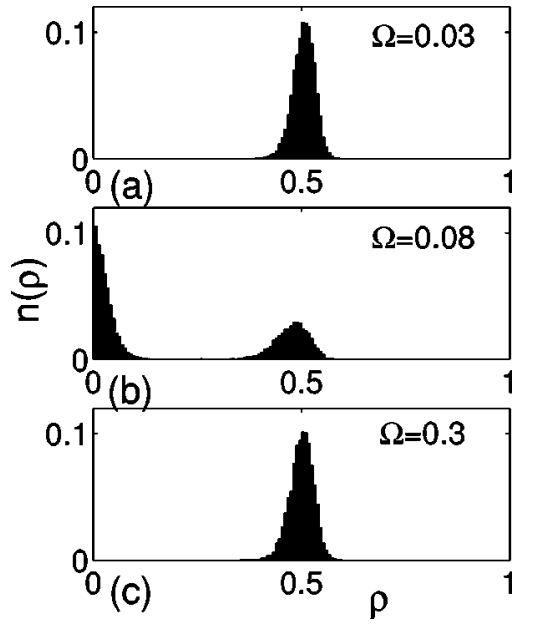

FIG. 12. The marginal probability distribution of the order parameter in the low (top panel), high (bottom panel), and intermediate (middle panel) driving force regimes for the same parameter values as in Fig. 11.

parameter values in the free energy used are $a_{1}=0.85, b_{1}$ $=5.8, c_{1}=8.0$, and for the strain field are $a_{2}=1.3644, b_{2}$ $=8.7105$, and $c_{2}=13.6740$. We study the time evolution of the system as a function of the drive $\Omega$ (for a fixed coupling parameter $\alpha$ ) and as a function of $\alpha$ for a fixed drive. We observe the following behavior.

\section{As a function of the drive $\Omega$}

Keeping $\alpha$ fixed at an optimum value $(\alpha=0.17)$, the drive has two thresholds:

(i) For $\Omega<\Omega_{1}$ and $\Omega>\Omega_{2}$, we find that the system always resides in the crystalline minimum $(\rho \neq 0)$. The time series of $\rho(t)$ is shown in Figs. 11(a,c) for a typical set of values $\Omega=0.03$ and $\Omega=0.3$, respectively. The corresponding probability distribution is peaked around the crystalline minimum, as can be seen from Figs. 12(a,c).

(ii) In an intermediate window of driving forces $\Omega_{1}<\Omega$ $<\Omega_{2}$, the system stochastically alternates between the liquid $(\rho=0)$ and the crystalline minimum. The time series corresponding to a typical value of $\Omega=0.08$ is shown in Fig. 11(b). The probability distribution has two peaks corresponding to these two minima. The time series of the order parameter [Fig. 11(b)] also shows that the persistence time of these two states are comparable (for optimum values of $\alpha$ ) very much like the time series of a system undergoing stochastic resonance. These results are similar to the results of the particle model where $F_{d}^{*}$ was varied for a fixed value of $\epsilon$.

\section{As a function of the coupling $\alpha$}

Here, we have kept the drive $\Omega$ at intermediate values. We find that the crystalline minimum is favored over the liquid one for low values of $\alpha$, whereas for high values the liquid minimum dominates. At intermediate values of $\alpha$, the system spends comparable durations in each state. As we increase $\alpha$ from small values, one finds that the system tends to spend increasingly more time in the liquid state and, eventually, at large values of $\alpha$ the liquid state is the preferred 


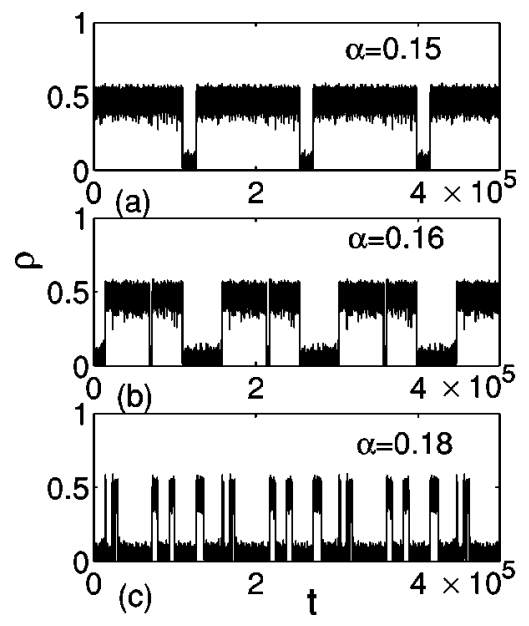

FIG. 13. The time series of the order parameter in the low (top panel), high (bottom panel), and intermediate (middle panel) values of the coupling parameter $\alpha$ for noise strength $D=7 \times 10^{-4}$ and driving force $\Omega=0.08$.

state. This is shown in Fig. 13 for three typical values of $\alpha$. (The numerical results are for $\Omega=0.08$, for which we observe the most prominent stochastic switching of the order parameter values between $\rho \neq 0$ and $\rho=0$.) This is also reflected in the probability distribution which has a more pronounced peak at the crystalline minimum at small $\alpha$ and at the liquid minimum at large $\alpha$. For intermediate values of $\alpha$ we find a bimodal distribution. Figure 14 shows the probability distributions for the parameter values of Fig. 13. This behavior is similar to the results obtained in the particle model by varying $\epsilon$ keeping $F_{d}^{*}$ fixed.

As mentioned, one dominant feature of SR is the enhancement of the signal-to-noise ratio (SNR) at an optimum value of the noise intensity. In order to check this, we have carried out long runs of the order of $10^{10}$ time steps. The power spectral density (PSD) of the time series has been calculated for various values of the noise intensity ranging from $D=6$

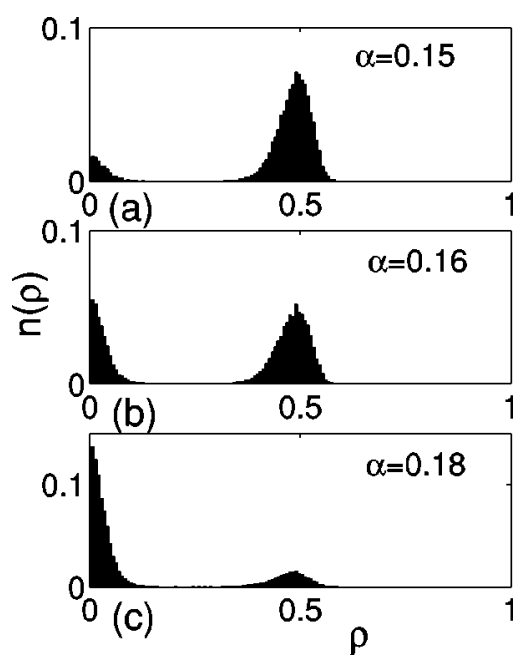

FIG. 14. The marginal probability distribution of the order parameter in the low (top panel), high (bottom panel), and intermediate (middle panel) $\alpha$, for the same parameter values as in Fig. 13.

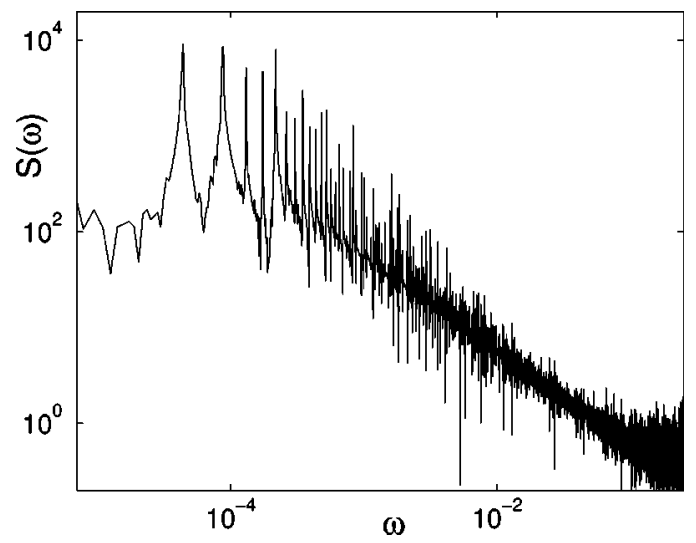

FIG. 15. The power spectrum of the time series of the order parameter $\rho$ for $\alpha=0.17, \Omega=0.08$, and $D=7.0 \times 10^{-4}$.

$\times 10^{-4}$ to $1.2 \times 10^{-3}$. It exhibits strong peaks at all integral values of the fundamental (unlike the symmetric bistable potential where only the odd harmonics are seen) due to the absence of any symmetry in $F(\rho, \theta)$. A plot of this is shown in Fig. 15 for a typical value of $D=7.0 \times 10^{-4}$. The signalto-noise ratio calculated from the power spectrum using the first peak for various values of $D$ is shown in Fig. 16. (Here we have used the conventional definition of SNR, namely, $10 \log \left[S_{\text {signal }}(\omega) / S_{\text {noise }}(\omega)\right]$.) As is clear from the figure, the maximum enhancement of the SNR is found to be around $D \sim 7.0 \times 10^{-4}$

As discussed above, we note that $\Omega$ and $\alpha$ of the reduced model take the roles of the drive $F_{d}^{*}$ and the interspecies interaction strength $\epsilon$ in the particle model, respectively. To make the parallel between these models more concrete, we have constructed the dynamical phase diagram in the $\alpha-\Omega$ plane shown in Fig. 17. (In constructing this diagram, we have taken the system to be a liquid state if it spends less than $2 \%$ of the time in the crystalline minimum, and correspondingly for the crystal.) Region I refers to the crystalline phase and region III, the reentrant crystalline phase. The melt-freeze cycles where the system alternates between crystal and liquid is shown as region II. In region II, for low values of the coupling parameter $\alpha$, the persistence of the ordered state is more than that for the disordered state, and

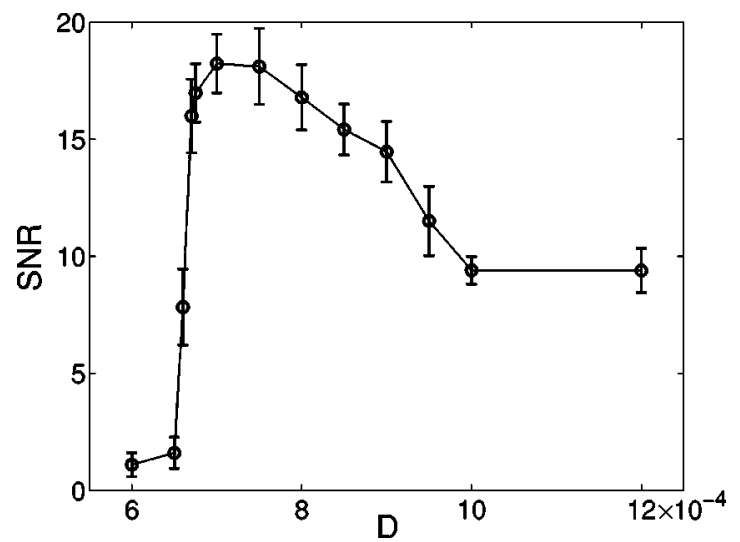

FIG. 16. The signal-to-noise ratio for the reduced model for parameter values $\alpha=0.17$ and $\Omega=0.08$. 


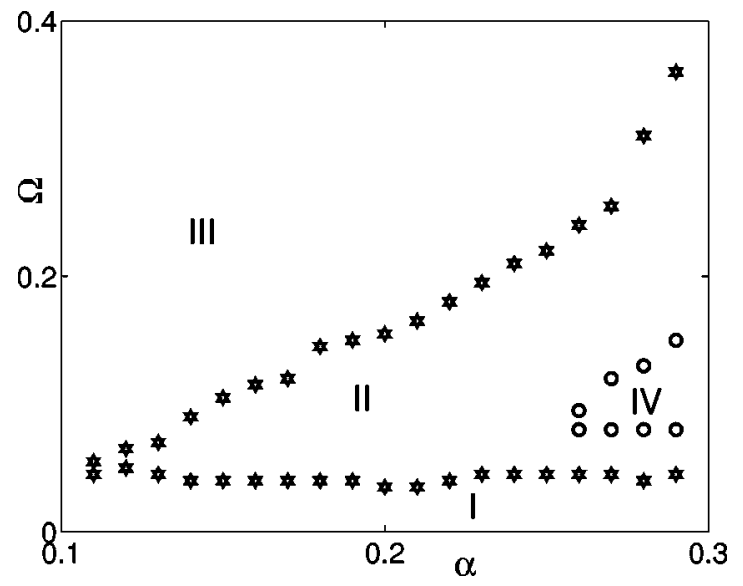

FIG. 17. The phase diagram of the reduced model in the $\alpha-\Omega$ plane. The system is crystalline in region I and region III represents the reentrant solid. In region II we observe the melt-freeze cycles. For high values, liquidlike region IV is seen again.

decreases as $\alpha$ is increased, eventually giving way to the liquidlike region IV for moderate values of $\Omega$. It is clear that this diagram is remarkably similar to the phase diagram of the particle model shown in Fig. 10, except for the region IV which could not be detected in the particle model within the longest runs carried out.

The physical picture of the reduced model is clear. As the initial state at zero shear rate is a crystalline state, by continuity arguments one should expect that at low shear rates the system should find the crystalline minimum favorable. At intermediate range of shear rates, the system develops another minimum at zero value of the order parameter $\rho$ corresponding to the melt state making the system bistable. When the shear rate is close to (and larger than) the critical value $\Omega_{c}$, under the action of noise, the systems make transitions from the crystalline minimum to the liquid minimum and vice versa. However, since the strain $\theta$ evolves in time, the system experiences an additional periodic modulation. We note here that this periodicity is not equal to $1 / \Omega$ as the strain variable moves on the $V(\theta)$ surface. Typical values of the induced periodicity estimated from deterministic version of Eqs. (8) and (9) for the optimum range of drive values is of the order of $10^{5}$ time units. When the induced periodicity is twice the Kramers escape rate [37], the system alternates between the two minima. Further, we note that as the two wells are not symmetric, in the presence of noise, the mean first passage times associated with the two wells will be different. Indeed, we find that as a function of $\Omega$, there is a range of $\Omega$ values $(0.065<\Omega<0.12)$ where the barrier between the crystalline minimum and maximum of the free energy, $F_{\text {saddle }}-F_{\text {crystal }}=\Delta F_{c}$, is much smaller than that between the liquidlike minimum and the maximum, $F_{\text {saddle }}$ $-F_{\text {liquid }}=\Delta F_{l}$. A plot of $\Delta F_{c}$ and $\Delta F_{l}$ is shown in Fig. 18. It is clear that $\Delta F_{l}$ is nearly constant as a function of $\Omega$ while $\Delta F_{c}$ goes through a minimum in the range of $\Omega$ $=0.065-0.12$. A simple order of magnitude calculation gives the Kramers rate [37] $T_{f}^{-1}=\left[\left(F_{\text {min }}^{\prime \prime}\left|F_{\text {saddle }}^{\prime \prime}\right|\right)^{1 / 2} / 2 \pi\right] \exp$ $-[\Delta F / D] \sim 10^{-5}$ which matches with the frequency range of the first harmonic seen in the PSD (see Fig. 15). We note

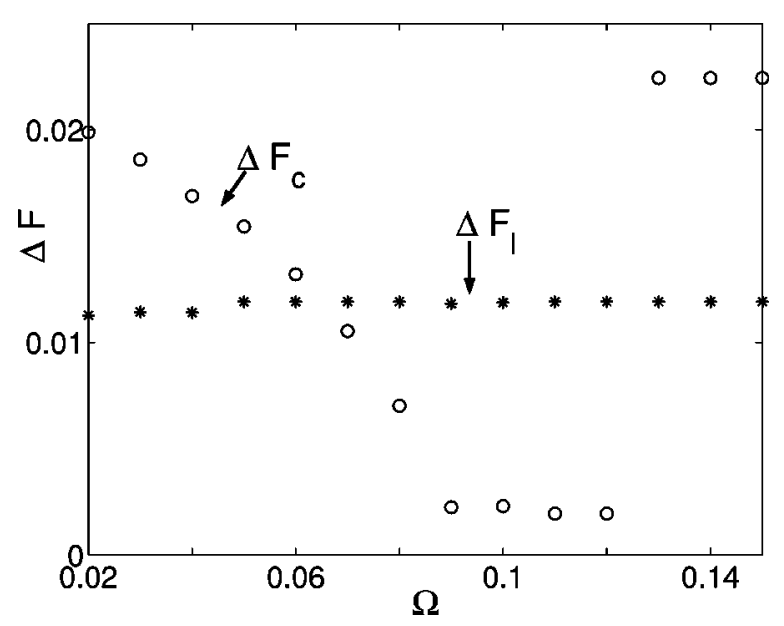

FIG. 18. $\Delta F_{c}$ and $\Delta F_{l}$ as a function of $\Omega$.

here that the first passage time for the system to cross the barrier, from the liquid state is much too large as the barrier $\Delta F_{l} \sim 0.01$. Thus, it is clear that the reduced model reproduces, qualitatively, most features of the particle model. The time series of $S(\mathbf{k}, t)$ in the particle model is very similar to that of $\rho(t)$ which we have demonstrated to have all the features of stochastic resonance. The regime showing stochastic resonance is more pronounced for optimum values of $\Omega$ and $\alpha$. In fact here, as in our particle model simulations, in the melt-freeze regime, for low values of the coupling parameter, the crystalline state is favored and for high values of the coupling parameter, the liquid state is favored, while in an intermediate regime they are roughly equal. The similarity of the reduced model with the particle model is well summarized by the phase diagram of the model in the $\Omega$ - $\alpha$ plane which is similar to that of the particle model in the $F_{d}^{*}-\epsilon$ plane.

\section{DISCUSSION}

In summary, we have studied the nonequilibrium statistical behavior of two adjacent monolayers sheared past each other, using Brownian dynamics simulations. For low and high driving forces, we obtain macroscopically ordered, steadily drifting states. In a suitable range of driving rates we see that the system switches between crystalline and liquidlike states. The residence times are nearly equal in an intermediate range of values of the inter-layer coupling. As we have seen, the interlayer coupling essentially determines the barrier each particle has to surmount in order keep pace with the applied drive. Our simulations show that the switching between the crystalline and liquidlike states maintains the spatial coherence (or the lack of it in the liquid state) and thus is an example of cooperative stochastic resonance. Although there is no external imposed time-periodic potential present in our system, its role is played by the drive which shears the two layers past each other. Thus, each moving layer provides a time-varying potential. To make the connection to SR, more concrete, we have introduced a reduced model for the dynamics of amplitude and phase of the crystalline order parameter [21] which mimics all features of the 
particle model apart from showing the main feature of SR, namely, the enhancement of signal-to-noise ratio for optimum values of noise strength. This relation between the particle model and the reduced model makes a convincing case that the melt-freeze cycles observed in the former are indeed a manifestation of stochastic resonance of a spatially extended [39] noisy interacting system subjected to a constant drive.

A few comments may be in order on the dynamics of the particle model. Although our model falls broadly into the class of shear driven systems which are known to display stick-slip behavior $[12,13,40]$ in most such cases where the system spends considerable time in the stuck phase and very little time in the slip phase. Experiments on confined fluids of few monolayers (a situation superficially similar to our model) and the corresponding molecular dynamics simulations $[12,13]$ show that the system also displays melt-freeze cycles with the crystalline phase persisting significantly much longer than the melt phase. From this point of view, the persistence dynamics we observe, with nearly equal residence times in crystalline and the liquid-like states, comes as a surprise and is clearly not conventional stick-slip motion. The reduced model has helped us to elucidate the connection to stochastic resonance apart from the similarity of the phase diagrams of the two models. However, it is clear that not all aspects of the particle model are mimicked by the reduced model. For instance, the feature observed for small interlayer coupling $\epsilon$, namely the smaller extent of order as seen by the small values of $S(\mathbf{k}, t)$ in the crystalline state (compared to larger values of $\epsilon$ ) and larger fluctuations is not seen in the reduced model. As explained earlier, this feature is a manybody effect appearing in a low barrier situation and therefore cannot be explained on the basis of the SR features of the reduced model valid only in the Kramers high barrier limit (even if one were to include appropriate spatial degrees of freedom).

Finally, let us comment on the experiments which can verify our simulations. We expect that this phenomenon should arise in adjacent crystal planes of sheared colloidal crystals. To study this effect in bulk sheared colloidal crystals would require not conventional scattering probes but something that focuses on an adjacent pair of crystal planes [41]. Alternately, we could look at two solid surfaces patterned with ordered copolymer $[42,43]$ or colloidal monolayers under relative shear. The colloids or copolymer adsorbed to the two surfaces must be of two different kinds, each having more affinity towards one of the surfaces. Another possibility would be to study electrokinetic motion of charged 2D confined binary colloids in the presence of a constant external electric field, generalizing the ideas of Ref. [20]. Center of mass measurements would be the same as those made for two species being driven in opposite directions.

\section{ACKNOWLEDGMENTS}

M.D. acknowledges CSIR, India for financial support, SERC, IISc for providing computational facilities and $\mathrm{C}$. Dasgupta, B. Chakrabarti, and C. Das for useful discussions.
[1] B. N. J. Persson, Sliding Friction: Physical Principles and Applications, Nanoscience and Technology Series (Springer, New York, 1998).

[2] M. O. Robbins and M. H. Muser, in Modern Tribology Handbook, edited by B. Bhushan (CRC Press, Boca Raton, FL, 2001), p. 717.

[3] L. Balents, M.C. Marchetti, and L. Radzihovsky, Phys. Rev. B 57, 7705 (1998).

[4] T. Giamarchi and P. Le Doussal, Phys. Rev. Lett. 76, 3408 (1996).

[5] J.B. Salmon, A. Colin, and D. Roux, Phys. Rev. E 66, 031505 (2002).

[6] D. Lopez et al., Phys. Rev. Lett. 82, 1277 (1999).

[7] J.A. Weiss, A.E. Larsen, and D.G. Grier, J. Chem. Phys. 109, 8659 (1998).

[8] T. Palberg and K. Streicher, Nature (London) 367, 51 (1994).

[9] A. Imhof, A. van Blaaderen, and J.K.G. Dhont, Langmuir 10, 3477 (1994).

[10] B.J. Ackerson and N.A. Clark, Phys. Rev. A 30, 906 (1984).

[11] H.M. Lindsay and P.M. Chaikin, J. Phys. (Paris), Colloq. 46, C3 (1985).

[12] J.N. Israelachvili, P.M. McGuiggan, and A.M. Homola, Science 240, 189 (1988).

[13] P.A. Thompson and M.O. Robbins, Science 250, 792 (1990).

[14] C. Drummond, J. Elezgaray, and P. Richetti, Europhys. Lett. 58, 503 (2002).
[15] O.M. Braun, A.R. Bishop, and J. Roder, Phys. Rev. Lett. 82, 3097 (1999); O.M. Braun and M. Peyrard, Phys. Rev. E 63, 046110 (2001)

[16] Ya. Frenkel and T. Kontorova, Phys. Z. Sowjetunion 13, 1 (1938).

[17] B.N.J. Persson, Phys. Rev. Lett. 71, 1212 (1993).

[18] E. Granato and S.C. Ying, Phys. Rev. B 59, 5154 (1999); Phys. Rev. Lett. 85, 5368 (2000)

[19] M. Das, S. Ramaswamy, and G. Ananthakrishna, Europhys. Lett. 60, 636 (2002).

[20] J. Dzubiella, G.P. Hoffmann, and H. Lowen, Phys. Rev. E 65, 021402 (2002).

[21] R. Lahiri and S. Ramaswamy, Phys. Rev. Lett. 73, 1043 (1994).

[22] R. Benzi, A. Sutera, and A. Vulpiani, J. Phys. A 14, L453 (1981); R. Benzi, G. Parisi, A. Sutera, and A. Vulpiani, Tellus 34, 10 (1982).

[23] C. Nicolis, Sol. Phys. 74, 473 (1981); C. Nicolis and G. Nicolis, Tellus 33, 225 (1981).

[24] L. Gammaitoni, P. Hanggi, P. Jung, and F. Marchesoni, Rev. Mod. Phys. 70, 223 (1998); V. Anishchenko, A. Neiman, F. Moss, and L. Schimansky-Geier, Phys. Usp. 42, 7 (1999).

[25] B. McNamara and K. Wiesenfeld, Phys. Rev. A 39, 4854 (1989).

[26] The local variance of the velocity is not, of course, a good measure of long-range correlations. To detect the latter we 
would need to measure the spatial correlation function of the drift velocity, which we have not done.

[27] A. Chowdhury, B.J. Ackerson, and N.A. Clark, Phys. Rev. Lett. 55, 833 (1985).

[28] J.L. Barrat and H. Xu, J. Phys.: Condens. Matter 2, 9445 (1990).

[29] C. Das and H.R. Krishnamurthy, Phys. Rev. B 58, R5889 (1998).

[30] E. Frey, D.R. Nelson, and L. Radzihovsky, Phys. Rev. Lett. 83, 2977 (1999).

[31] B.J. Ackerson and P.N. Pusey, Phys. Rev. Lett. 61, 1033 (1988).

[32] H. Komatsugawa and S. Nose, Phys. Rev. E 51, 5944 (1995).

[33] M.O. Robbins and M.J. Stevens, Phys. Rev. E 48, 3778 (1993).

[34] A. Onuki, J. Phys.: Condens. Matter 15, S891 (2003).

[35] B.J. Ackerson and N.A. Clark, Phys. Rev. Lett. 46, 123 (1981).

[36] Note that the equations of motion do not have a factor $\rho$ mul- tiplying $\dot{\theta}$, so that even though $\theta$ is periodic, we have a planar metric.

[37] H.A. Kramers, Physica (Utrecht) 7, 284 (1940).

[38] P. Hanggi, P. Talkner, and M. Borkovec, Rev. Mod. Phys. 62, 251 (1990).

[39] J.M.G. Vilar and J.M. Rubi, Phys. Rev. Lett. 78, 2886 (1997), show that a conventional oscillating-field modulation of the Swift-Hohenberg equation for a continuous transition to a layered state also shows spatiotemporal stochastic resonance.

[40] L.S. Aranson, L.S. Tsimring, and V.M. Vinokur, Phys. Rev. B 65, 125402 (2002).

[41] D.G. Grier, Curr. Opin. Colloid Interface Sci. 2, 264 (1997).

[42] J. Klein, D. Perahia, and S. Warburg, Nature (London) 352, 143 (1991).

[43] C. Robelin, F.P. Duval, P. Richetti, and G.G. Warr, Langmuir 18, 1634 (2002). 